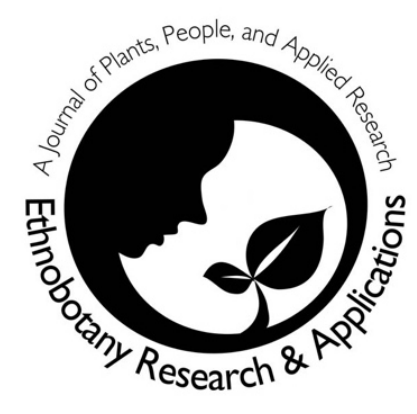

\title{
Traditional knowledge and use of wild edible plants in Sidi Bennour region (Central Morocco)
}

\author{
Abdelghani Aboukhalaf, Manal Tbatou, Adil Kalili, Kaoutar \\ Naciri, Sara Moujabbir, Khadija Sahel, João Miguel Rocha \\ and Rekia Belahsen
}

\begin{abstract}
Correspondence
Abdelghani Aboukhalaf ${ }^{1}$, Manal Tbatou ${ }^{1}$, Adil Kalili ${ }^{1}$, Kaoutar Naciri ${ }^{1}$, Sara Moujabbir ${ }^{1}$, Khadija Sahel ${ }^{1}$, João Miguel Rocha ${ }^{2}$ and Rekia Belahsen ${ }^{1}$

${ }^{1}$ Laboratory of Biotechnology, Biochemistry and Nutrition, Training and Research Unit on Nutrition and Food Sciences, Department of Biology, Faculty of Sciences, Chouaib Doukkali University, El Jadida, 24000, Morocco ${ }^{2}$ Laboratory for Process Engineering, Environment, Biotechnology and Energy, Faculty of Engineering, University of Porto, Rua Dr. Roberto Frias, 4200-465 Porto, Portugal

*Corresponding Author: b.rekia@gmail.com
\end{abstract}

Ethnobotany Research and Applications 23:11 (2022)

\section{Research}

\begin{abstract}
Background: This study aimed to protect the knowledge related to the traditional uses of wild plant resources which constituted untapped potential as dietary supplements and therapeutic products
\end{abstract}

Methods: An ethnobotanical survey based on the Semi-Structured Interview method was carried out among the Sidi Bennour region from February 2019 to February 2020.

Results: The results showed that a total of 56 plant species representing 56 genera and 27 families were used to make different food dishes and in the treatment of various diseases. The most cited wild edible plants (WEPs) families were Asteraceae (16\%). The leaves (38\%) and stems (32\%) were the most parts commonly used in food. Four utilization categories were cited, vegetables, for seasoning, as a drink, and Other (plants used to decorate or flavor traditional dishes). The use of these WEPs as vegetables was the most cited mode of consumption (37.5\%) by the local population. The leaves were the most commonly used part (35\%) for medicinal uses of WEPs. Decoction (27.27\%) was the most common method of preparing traditional medicines. The majority of preparations were administered orally (80.25\%). The study results showed also that local people have sufficient information on the safe use of WEPs.

Conclusions: The present study demonstrated that the people of the Sidi Bennour region, hold rich traditional knowledge of a large number of WEPs, however, the study population underlined the sharp decline in the consumption of most of the species recorded consequently the detailed documentation may effectively prevent knowledge loss through time.

Keywords: ethnobotanical survey, Wild edible plants, relative frequency index of citation, phytotherapy, beqoula, Sidi Bennour, Morocco

\section{Background}

Wild Edible Plants (WEP) are defined as plant species that are neither cultivated nor managed, but accessible from various natural types of natural vegetation and used as food (Bhatia et al. 2018), These plants have always been an important part of the human diet and were the basis of preserving human survival for thousands of years before 
the development of agriculture. With the development of agricultural methods, the process of domestication of some of these plants began to produce sufficient amounts of food throughout the year instead of being limited to seasons and climatic conditions. The status of WEPs began to decline with the current dependence of the agricultural system on productive crops, causing a sharp decline in varieties and the level of agricultural diversification.

Morocco benefits from one of the richest plant diversities in the Mediterranean basin (Nassif \& Tanji 2013), because of its exceptional position, with the Mediterranean Sea in the north, the Atlantic Ocean in the west, and the Sahara Desert in the south and east. Wild Edible Plants are part of this diversity. In Morocco, the tradition of using WEPs continues to be part of the dietary habits of local populations, particularly those in rural areas (Aboukhalaf et al, 2020). Most ethnobotanical studies in Morocco focus on medicinal and aromatic plants. By contrast, ethnobotanical research on WEPs has been somewhat neglected with only a few reports documenting the traditional use of these plants, (Hmamouchi 1997, Nassif \& Tanji 2013, Powel et al 2014, Tbatou et al. 2016a, Tbatou et al. 2016b). hence the need for further systematic studies to collect data in this regard. In addition, WEPs are an alternative source of income for many populations (Bhatia et al. 2018, Samant \& Dhar 1997, Shrestha \& Dhillion 2006). They could play an important role in food insecurity. They can be used to fight against malnutrition given their high content in nutrients and vitamins (Burlingame 2000, Glew et al. 2005, Sarfo et al. 2020). Indeed, the fact that WEPs are rich sources of bioactive molecules is now argued (Aboukhalaf et al. 2020, Morales et al. 2014). In addition, these plants are valuable genetic resources that can be used for the development of new crop species (Shrestha $\&$ Dhillion 2006).

However, the tradition of using WEPs and the associated knowledge decline with urbanization, modernization of traditions, industrialization, the temptation of fast food, deforestation, and the lack of interest in the young generation, etc. (Bhatia et al. 2014, Bhatia et al. 2018, Łuczaj et al. 2013, Tbatou et al. 2016b), It is therefore essential to document this valuable traditional knowledge before it is lost. This study aims to report the various wild edible plants of the Sidi Bennour region and the traditional practices related to their use, to evaluate and compare the cultural importance of these plants in the local population and, finally to assess and compare the trend of exploitation of these plants in the study area.

\section{Materials and Methods}

\section{Study area}

Morocco, bounded by the Atlantic Ocean to the west and the Province of El-Jadida in the northwest (DRACS 2018). The province climate is semi-arid, with a humid temperate winter and generally hot and dry summer (HCP 2014), The dominant soil types are Vertisols (30\%), chestnut and red (25\%) and sandy loam (25\%) (DRACS 2018). The average annual rainfall is around $300 \mathrm{~mm}$ per year (DRACS 2018). The Province of Sidi Bennour, which covers an area of $300.733 \mathrm{~km}^{2}$, encompasses 25 municipalities, 2 urban and 23 rural (DRACS 2018). According to the 2014 General Population and Housing Census, the Province population was 460.000 inhabitants, with an urbanization rate not exceeding 17\% (HCP 2014). The economic activity in this province depends largely on the agricultural sector; it is one of Morocco's leading suppliers of red meat, milk, sugar, and other agriculture products (DRACS 2018) (Fig. 1).

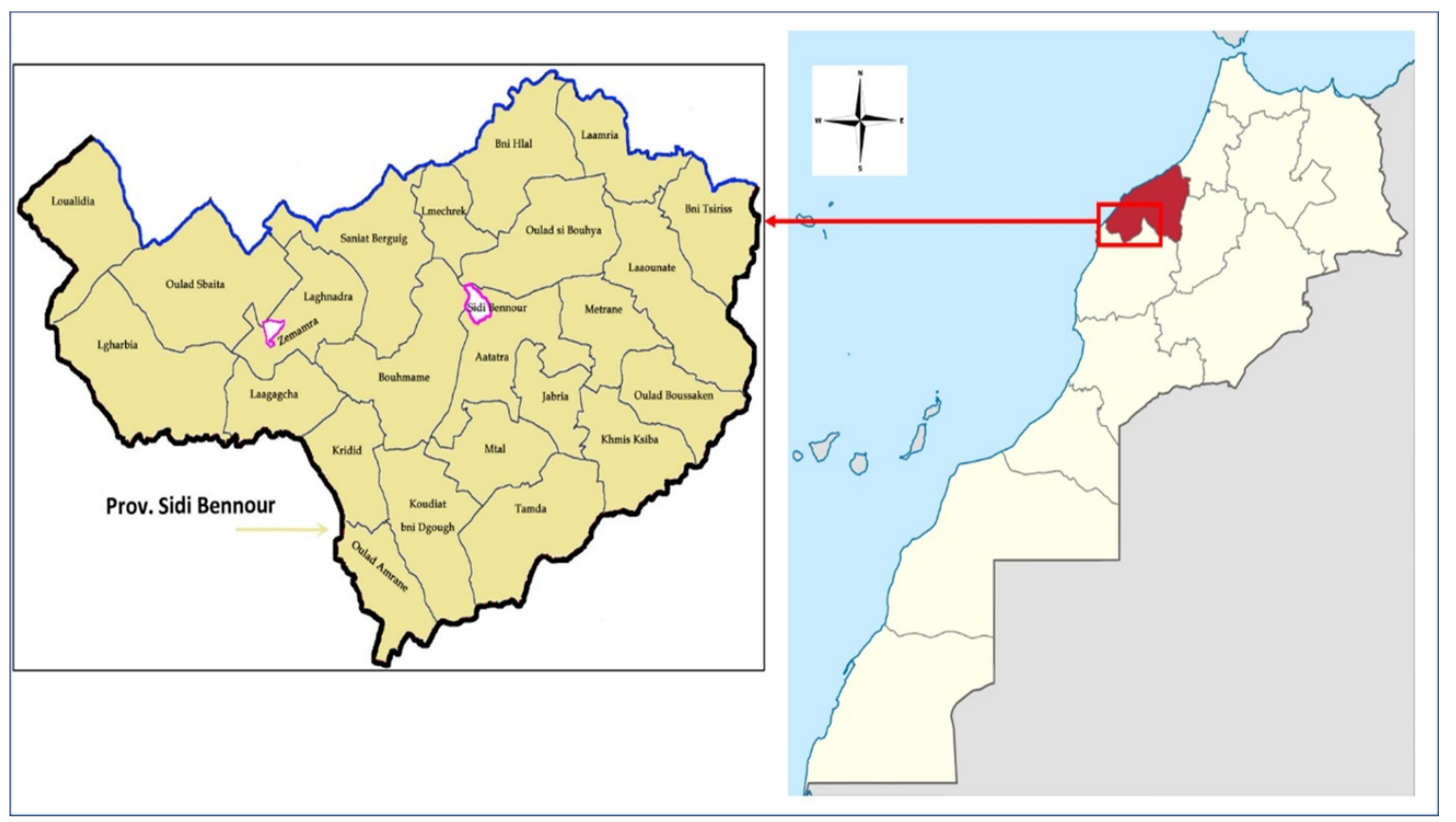


Figure 1. Location of the study area (DRACS 2018)

\section{Data collection tools and procedures}

The ethnobotanical survey was conducted from February 2019 to February 2020, using a questionnaire to collect information on the socio-demographic profile of the people surveyed (age, sex, academic level, etc.), information details on the traditional knowledge of WEPs and their different types of use (food, medicinal, etc.), as well as the dynamics of their use over time. For each species mentioned, the local name, the part used, and the preparation method were recorded. For medicinal uses, the mode of administration and the diseases treated were also recorded. A list with photos was also prepared for the WEPs used as a source of food or as medicine in areas close to the study area to facilitate the interview (Vitalini et al. 2013). Only plants mentioned by 3 or more respondents were taken into account (Ali-Shtayeh et al. 2008). As our objective was to collect as much information as possible on the WEPs used traditionally, we relied for the selection of informants who were knowledgeable about wild edibles plants using the purposive sampling technique (sukenti et al. 2016) to get the required important information about these plants. The key informants were selected based on recommendations of local dwellers and local guides, the majority of them were females and above 40 years old. Before conducting interviews, the aim of the study was explained clearly, and informants were asked for their consent.. The sample size surveyed was 200 people. The surveys were based on the Semi-Structured Interview method (Martin 1995). The approach of the interviewed population was based on dialogue in the local language. The time spent on each interview was approximately 40 minutes to an hour.

\section{Plant identification}

Field visits were organized with the help of informants to collect specimens of plants for identification and deposit in a herbarium. The taxonomic identification of the species was carried out by comparing our samples with those kept in the herbarium of our laboratory (biotechnology, biochemistry, and nutrition laboratory of Chouaib Doukkali University) and with the help of a botanist. Scientific names were reviewed online using the Plant List Database (www.theplantlist.org) and some literature references of botanical data on Morocco (Bellakhdar et al. 1991, Bellakhdar 1997, Tanji 2005, Valdés 2002). When discrepancies in taxonomy were found, accepted names by the Plant List Database were used.

\section{Data analysis}

The data recorded on the survey forms were entered into a spreadsheet and presented in graphs and tables. The data analysis was carried out using simple descriptive statistics methods. Furthermore, to assess the local importance of each species and to determine the most preferred species, the Relative Citation Frequency Index (RFC) was used calculated using the following formula (Tardío and Pardo-de-Santayana 2008)

$$
\boldsymbol{R F C}=\frac{\mathbf{F C}}{\mathbf{N}}(0 \leq \mathrm{RFC} \leq 1) \text { where: }
$$

$\mathrm{FC}=$ Number of informants mentioning the uses of the species.

$\mathrm{N}=$ Total number of informants in the study.

\section{Results and Discussion}

\section{Sociodemographic characteristics}

In this work, most selected informants were over $40 \mathrm{yr}$ old, , these informants collect plants for medicinal and food purposes more frequently and still preserve the traditional knowledge, Similar studies also found that traditional knowledge increases with age and length of residence in a region (Guimbo et al. 2011, Vitalini et al. 2013, Mugisha et al 2014). Indeed, Tbatou et al (2016b), Silva et al (2011), and Rangel et al (2010) confirmed that practical knowledge, or skills for identifying wild plants, increased with age as a result of long experience accumulated and passed down from generation to generation.

Most of those selected for the survey were women (86\%) (Table 1), as several previous studies reported that women knew more about plants for household use, including food and medicinal plants (Ayantunde et al. 2008, Silva et al. 2011). Moreover, Nassif et Tanji (2013) have shown that it is women who know most about edible plants, when to pick them and how to turn them into good foods throughout the year.

In addition, the majority of the population selected for the present survey (95\%) (Table 1) lived in rural areas. The use of wild plants for human consumption is rared in Moroccan urban areas (Nassif \& Tanji 2013). The majority of the study population was not educated (89\%) (Table 1), indicating that the origins of their knowledge about the use of wild edible plants came from ancestors or from personal experiences. 
Table 1. Socio-demographic characteristics of the respondents

\begin{tabular}{ccc}
\hline Categories & Frequency & Percentage \\
Age & & \\
40 to 59 years & 86 & $43 \%$ \\
60 to 80 years & 98 & $49 \%$ \\
$>80$ years & 16 & $8 \%$ \\
Gender & 172 & $86 \%$ \\
F & 28 & $14 \%$ \\
M & & \\
Family situation & 8 & $4 \%$ \\
Single & 146 & $83 \%$ \\
Married & 2 & $1 \%$ \\
Divorced & 44 & $22 \%$ \\
Widower & 178 & $89 \%$ \\
Study level & 6 & $3 \%$ \\
Illiterate & 12 & $6 \%$ \\
Koranic & 4 & $2 \%$ \\
Primary & & \\
Secondary & 190 & $95 \%$ \\
Area of residence & 10 & $5 \%$ \\
Rural & & \\
Urban & & \\
\hline
\end{tabular}

\section{Diversity of wild edible plants}

We registered 56 species of wild edible plants in the study area. Of these species, 13 had only food use. In contrast, the other 43 species have both food and medicinal uses. The latter belong to 56 genera and 27 families (Table 2). Among the most cited botanical families the Asteraceae come in first with 10 species (17.86\%). The family is also the largest in the Moroccan vascular flora with 550 species, 131 of which are endemic (Fennane \& Tattou 2008). Apiaceae are in second place with 7 species (12.5\%), Lamiaceae with 4 in third (7.14\%), followed by Fabaceae, Amaranthaceae, Caryophyllaceae and Brassicaceae with 3 species each (5.36\%). The remaining families were represented by one or two species (Fig. 2). The most cited WEPs with at least one use mentioned by more than $75 \%$ of informants, were in descending order: Malva multiflora (Cav.) Soldano, Banfi \& Galasso. (RFC = 0.99), Portulaca oleracea L. (RFC $=0.95)$, Astragalus boeticus L. (RFC $=0.93)$, Chenopodium murale L. (RFC $=0.84)$, Ridolfia segetum $(\mathrm{L}$.) Moris (RFC $=0.82)$, Scolymus hispanicus L. (RFC $=0.80)$, Calendula stellata Cav. $(\mathrm{RFC}=0.76)$ and Oxalis pes-caprae L. (RFC $=0.75)$. Ethnobotanical studies carried out in other regions of Morocco have already reported $S$. hispanicus $\mathrm{L}, C$. murale $\mathrm{L}$ and Foeniculum vulgare Mill. as the most cited wild food plants in the region of the present study (Tbatou et al. 2016b). Other authors have also mentioned $P$. oleracea $\mathrm{L}$ as being a plant often cited in Morocco (Hadjichambis et al. 2008) (Table 2).

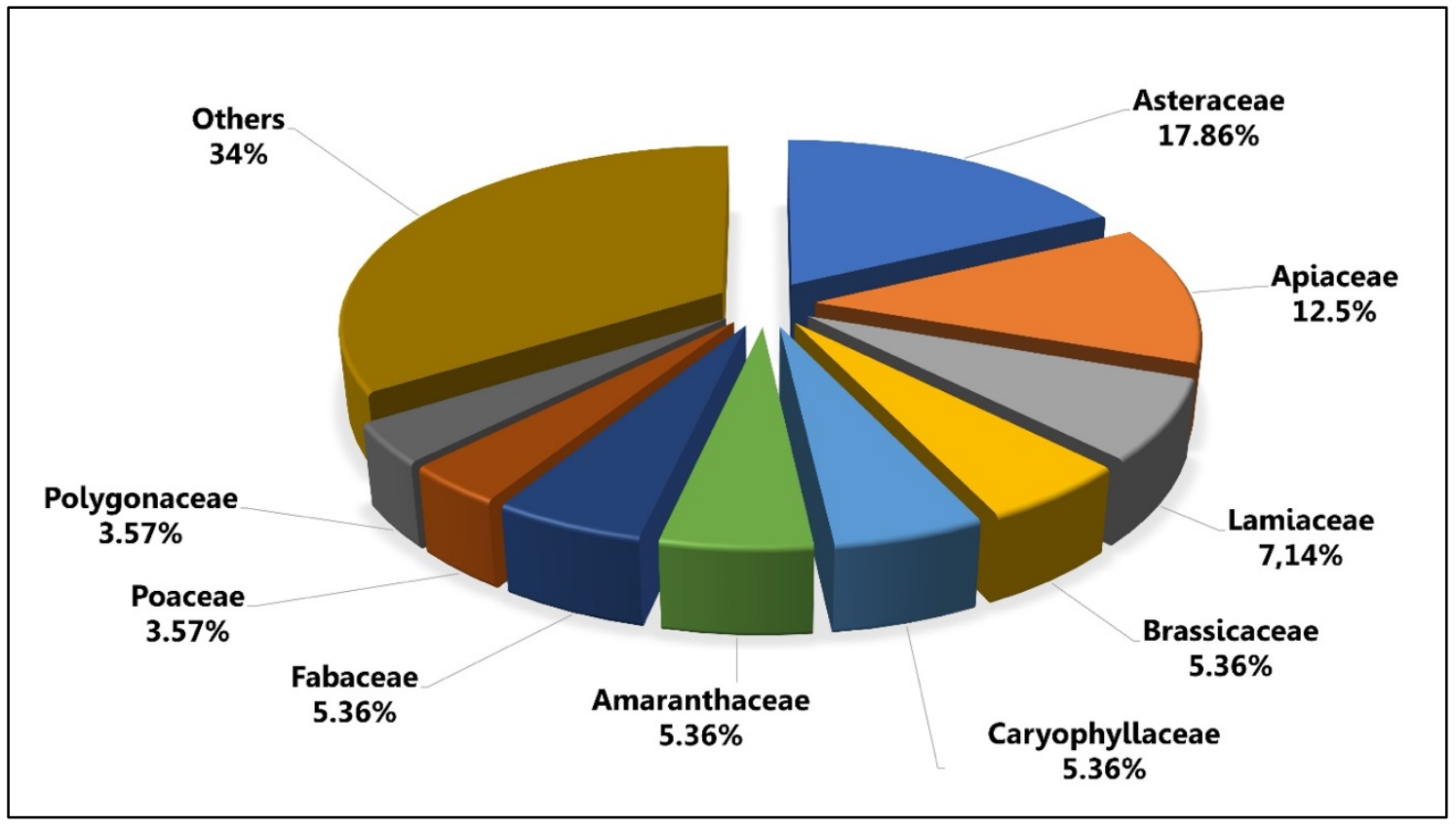

Figure 2. Most representative families. 
Table 2. List of wild edible plants used in the region of Sidi Bennour

\begin{tabular}{|c|c|c|c|c|c|c|c|}
\hline Family & $\begin{array}{l}\text { Species } \\
\text { (Voucher specimen) }\end{array}$ & Local name & $\begin{array}{l}\text { Type of } \\
\text { use }\end{array}$ & Purpose of use & Part used & Method of preparation & $\begin{array}{l}\text { Number } \\
\text { of } \\
\text { citations }\end{array}$ \\
\hline \multirow[b]{2}{*}{ Amaranthaceae } & \multirow{2}{*}{$\begin{array}{l}\text { Beta macrocarpa Guss } \\
\text { (BM7) }\end{array}$} & \multirow[b]{2}{*}{ Boumselli } & \multirow[b]{2}{*}{$\mathrm{F}$} & & Tender stems & Raw (Snacks) & 0.16 \\
\hline & & & & & $\begin{array}{l}\text { Young leaves, } \\
\text { tender stems }\end{array}$ & Used to prepare beqoula dish (Vegetables) & 0.14 \\
\hline \multirow{7}{*}{ Amaranthaceae } & \multirow{7}{*}{$\begin{array}{l}\text { Dysphania ambrosioides } \\
\text { (L.) Mosyakin \& Clemants } \\
\text { (MZ5) }\end{array}$} & \multirow{7}{*}{ Mkhinza } & \multirow{4}{*}{$\mathrm{F}$} & & \multirow{3}{*}{ Leaves } & Used to prepare beqoula dish (Vegetables) & 0.08 \\
\hline & & & & & & $\begin{array}{l}\text { Chopped leaves are added to the bread dough } \\
\text { (Other: perfuming) }\end{array}$ & 0.29 \\
\hline & & & & & & Cooked in the soup (Other: perfuming) & 0.66 \\
\hline & & & & \multirow{4}{*}{ Fever } & \multirow{4}{*}{ Leaves } & Oral: mixed with orange juice & 0.93 \\
\hline & & & \multirow{3}{*}{ Me } & & & Oral: infusion & 0.75 \\
\hline & & & & & & Oral: mixed with the onion juice & 0.68 \\
\hline & & & & & & Skin application: poultice & 0.28 \\
\hline \multirow{4}{*}{ Amaranthaceae } & \multirow{4}{*}{$\begin{array}{l}\text { Chenopodium murale L } \\
\text { (BR5) }\end{array}$} & \multirow{4}{*}{ Berremram } & \multirow[t]{2}{*}{$\mathrm{F}$} & & $\begin{array}{l}\text { Young leaves, } \\
\text { tender stems }\end{array}$ & Used to prepare beqoula dish (Vegetables) & 0.84 \\
\hline & & & & & Seeds & Cooked in the soup (Other: perfuming) & 0.25 \\
\hline & & & \multirow{2}{*}{$\mathrm{Me}$} & Rheumatism & Leaves & Oral: infusion & 0.09 \\
\hline & & & & Cold & Leaves, stems & Inhalation: fumigation & 0.11 \\
\hline \multirow{8}{*}{ Amaryllidaceae. } & \multirow{8}{*}{$\begin{array}{l}\text { Allium roseum } \mathrm{L} \\
\text { (KR12) }\end{array}$} & \multirow{8}{*}{ Korrita } & \multirow{2}{*}{$\mathrm{F}$} & & \multirow{2}{*}{ Bulbs } & used as a vegetable in corn couscous (Vegetables) & 0.15 \\
\hline & & & & & & Raw (Snacks) & 0.09 \\
\hline & & & \multirow{6}{*}{$\mathrm{Me}$} & Cold,jaundice & \multirow{3}{*}{ Bulbs } & Oral: decoction & 0.08 \\
\hline & & & & Rheumatism & & Skin application: poultice & 0.06 \\
\hline & & & & Stomach disorders & & Oral: raw & 0.18 \\
\hline & & & & \multirow{2}{*}{ Jaundice } & \multirow{3}{*}{ Leaves, stems } & Oral: fumigation & 0.03 \\
\hline & & & & & & Oral: cooked & 0.12 \\
\hline & & & & Cold & & Inhalation: fumigation & 0.05 \\
\hline \multirow{3}{*}{ Apiaceae } & \multirow{3}{*}{$\begin{array}{l}\text { Ammi majus L } \\
\text { (TR2) }\end{array}$} & \multirow{3}{*}{ Trilal } & & & & Spices (Seasoning) & 0.06 \\
\hline & & & $\mathrm{F}$ & & Leaves, stems & Powder mixed with bread flour (Other: perfuming) & 0.07 \\
\hline & & & $\mathrm{Me}$ & Fractures & Whole plant & Skin application: poultice & 0.08 \\
\hline & & & & & & Porridge in soup (Other: perfuming) & 0.19 \\
\hline & & & $\mathrm{F}$ & & Roots & Raw (Snacks) & \\
\hline & & & & & & Spices (Seasoning) & 0.22 \\
\hline & Elaeoselinum asclepium & & & Fever, headache & Roots & Oral: decoction & 0.13 \\
\hline Apiaceae & $\begin{array}{l}\text { (L.) Bertol } \\
(\mathrm{KH} 13)\end{array}$ & Kolikha & & $\begin{array}{l}\text { Postpartum pain, } \\
\text { infertility }\end{array}$ & Leaves, stems & Oral: cooked with bean or lentils & 0.15 \\
\hline & & & $\mathrm{Me}$ & Stomach disorders & & Oral: decoction & 0.21 \\
\hline & & & & $\begin{array}{l}\text { Cold, back pain, } \\
\text { improve sexual ability }\end{array}$ & & Oral: decoction & 0.16 \\
\hline
\end{tabular}




\section{Ethnobotany Research and Applications}

\begin{tabular}{|c|c|c|c|c|c|c|c|}
\hline \multirow{7}{*}{ Apiaceae } & \multirow{7}{*}{$\begin{array}{l}\text { Foeniculum vulgare Mill } \\
\text { (BS22) }\end{array}$} & \multirow{7}{*}{$\begin{array}{l}\text { Besbas } \\
\text { beldi }\end{array}$} & \multirow{2}{*}{$\mathrm{F}$} & & $\begin{array}{l}\text { Young leaves and } \\
\text { tender stems }\end{array}$ & Used to prepare beqoula dish (Vegetables) & 0.69 \\
\hline & & & & & & & \\
\hline & & & \multirow{5}{*}{$\mathrm{Me}$} & laundice anemia & Aerial narts & Oral- (onked nowder & 0.13 \\
\hline & & & & & Jeaves stems & & 0.20 \\
\hline & & & & Digestive disorders & Roots & Oral: decoction, raw & 0.21 \\
\hline & & & & & Roots, seeds & Oral: powder & 0.14 \\
\hline & & & & Anemia & Tender stems & Oral: raw & 0.31 \\
\hline \multirow{6}{*}{ Apiaceae } & \multirow{6}{*}{$\begin{array}{l}\text { Ridolfia segetum (L.) Moris } \\
\text { (TH12) }\end{array}$} & \multirow{6}{*}{$\begin{array}{l}\text { Tebch } \\
\text { Chebt }\end{array}$} & \multirow{3}{*}{\multicolumn{2}{|c|}{$\mathrm{F}$}} & \multirow{3}{*}{$\begin{array}{l}\text { Young leaves, } \\
\text { tender stems } \\
\text { Roots }\end{array}$} & Used to prepare beqoula dish (Vegetables) & 0.37 \\
\hline & & & & & & Raw (Snacks) & 0.82 \\
\hline & & & & & & Spices (Seasoning) & 0.19 \\
\hline & & & \multirow{3}{*}{$\mathrm{Me}$} & Anemia, cancer & \multirow{2}{*}{ Leaves and stems } & Oral: infusion, raw, cooked & 0.56 \\
\hline & & & & Jaundice & & \multirow{2}{*}{ Oral: cooked } & 0.14 \\
\hline & & & & Rheumatism & Leaves, seeds & & 0.19 \\
\hline \multirow{3}{*}{ Apiaceae } & \multirow{3}{*}{$\begin{array}{l}\text { Scandix pecten-veneris } \mathrm{L} \\
\text { (MT6) }\end{array}$} & \multirow{3}{*}{ Mechita } & \multirow{2}{*}{\multicolumn{2}{|c|}{$\mathrm{F}$}} & Tender stems & Used to prepare beqoula dish (Vegetables) & 0.09 \\
\hline & & & & & leaves & Spices (Seasoning) & 0.08 \\
\hline & & & $\mathrm{Me}$ & $\begin{array}{l}\text { Rheumatism, stomach } \\
\text { disorders, cold }\end{array}$ & Leaves & Oral: decoction & 0.06 \\
\hline \multirow{3}{*}{ Apiaceae } & \multirow{3}{*}{$\begin{array}{l}\text { Smyrnium olusatrum } L \\
\text { (HR70) }\end{array}$} & \multirow{3}{*}{ Elhiyar } & \multirow{2}{*}{\multicolumn{2}{|c|}{ a }} & \multirow{2}{*}{$\begin{array}{l}\text { Young leaves, } \\
\text { tender stems }\end{array}$} & Raw (Snacks) & 0.07 \\
\hline & & & & & & $\begin{array}{l}\text { Powder mixed with the bread flour (Other: } \\
\text { perfuming) }\end{array}$ & 0.05 \\
\hline & & & $\mathrm{Me}$ & Wound healing & Roots & Skin application: powder & 0.12 \\
\hline \multirow{3}{*}{ Apiaceae } & \multirow{3}{*}{$\begin{array}{l}\text { Thapsia villosa } \mathrm{L} \\
\text { (AB35) }\end{array}$} & \multirow{3}{*}{ Abo } & $\mathrm{F}$ & & Roots & Cooked in couscous (Other: food decoration) & 0.29 \\
\hline & & & \multirow{2}{*}{$\mathrm{Me}$} & Diabetes & Leaves, stems & Oral: decoction & 0.06 \\
\hline & & & & Stomach disorders & Roots & Oral: decoction & 0.1 \\
\hline \multirow{4}{*}{ Araceae } & \multirow{4}{*}{$\begin{array}{l}\text { Arisarum vulgare } \\
\text { O.Targ.Tozz } \\
\text { (YN3) }\end{array}$} & \multirow{4}{*}{ Yarni } & \multirow{4}{*}{$\mathrm{F}$} & & \multirow{4}{*}{ Bulbs } & $\begin{array}{l}\text { cooked in water for } 24 \text { hours to remove its toxicity } \\
\text { (Vegetables) }\end{array}$ & 0.49 \\
\hline & & & & & & $\begin{array}{l}\text { used as flour for the preparation of bread (Staple } \\
\text { foods) }\end{array}$ & 0.31 \\
\hline & & & & & & $\begin{array}{l}\text { Dry, grind the plant then put it in the couscoussier } \\
\text { and steam it seven times (Vegetables) }\end{array}$ & 0.18 \\
\hline & & & & & & Cooked in porridge (Vegetables) & 0.09 \\
\hline
\end{tabular}


Ethnobotany Research and Applications

\begin{tabular}{|c|c|c|c|c|c|c|c|}
\hline \multirow{3}{*}{ Asparagaceae } & \multirow{3}{*}{$\begin{array}{l}\text { Asparagus acutifolius L } \\
\text { (SK12) }\end{array}$} & \multirow{3}{*}{ Sekoum } & \multirow{2}{*}{\multicolumn{2}{|c|}{$\mathrm{F}$}} & \multirow{3}{*}{$\begin{array}{l}\text { Young shoot } \\
\text { Tender stems } \\
\text { Roots }\end{array}$} & \multirow{2}{*}{$\begin{array}{l}\text { cooked in broth (Vegetables) } \\
\text { Used to prepare beqoula dish (Vegetables) }\end{array}$} & \multirow{2}{*}{$\begin{array}{ll}0.28 \\
0.14 \\
\end{array}$} \\
\hline & & & & & & & \\
\hline & & & $\mathrm{Me}$ & $\begin{array}{l}\text { Intestinal disorders, } \\
\text { vermifuge, hepatitis }\end{array}$ & & Oral: decoction, juice & 0.16 \\
\hline \multirow{3}{*}{ Asteraceae } & \multirow{3}{*}{$\begin{array}{l}\text { Anacyclus maroccanus } \\
\text { (Ball) Ball } \\
\text { (RN13) }\end{array}$} & \multirow{3}{*}{ Rabiana } & $\mathrm{F}$ & & Leaves & Spices (Seasoning) & 0.29 \\
\hline & & & \multirow{2}{*}{ Me } & Cold, fever, redness & \multirow{2}{*}{ Flowers } & \multirow{2}{*}{ Oral: infusion } & 0.18 \\
\hline & & & & $\begin{array}{l}\text { Sedative, digestive } \\
\text { disorders }\end{array}$ & & & 0.09 \\
\hline \multirow{5}{*}{ Asteraceae } & \multirow{5}{*}{$\begin{array}{l}\text { Calendula stellata Cav } \\
\text { (JM1) }\end{array}$} & \multirow{5}{*}{$\begin{array}{l}\text { Ejjamra, } \\
\text { Hamra- } \\
\text { jamra }\end{array}$} & \multirow{5}{*}{$\mathrm{F}$} & & $\begin{array}{l}\text { Young leaves, } \\
\text { Tender stems }\end{array}$ & Used to prepare beqoula dish (Vegetables) & 0.76 \\
\hline & & & & & Flowers & Spices (Seasoning) & 0.21 \\
\hline & & & & & Tender stems & Raw (Snacks) & 0.22 \\
\hline & & & & & Flowers & $\begin{array}{l}\text { Used in the preparation of couscous (Other: } \\
\text { perfuming) }\end{array}$ & 0.16 \\
\hline & & & & & Roots & $\begin{array}{l}\text { Powder mixed with the bread flour (Other: } \\
\text { perfuming) }\end{array}$ & 0.63 \\
\hline \multirow{6}{*}{ Asteraceae } & \multirow{6}{*}{$\begin{array}{l}\text { Carthamus lanatus L } \\
\text { (AS45) }\end{array}$} & \multirow{6}{*}{ Asfar } & \multirow{3}{*}{$\mathrm{F}$} & & Roots & Spices (Seasoning) & 0.11 \\
\hline & & & & & leaves, stems & Cooked with the snail (Other: perfuming) & 0.08 \\
\hline & & & & & Flowers & $\begin{array}{l}\text { used in the preparation of margarine (Other: } \\
\text { perfuming) }\end{array}$ & 0.09 \\
\hline & & & \multirow{5}{*}{ Me } & Redness, anemia & Whole plant & Oral: cooked in bean or lentil soup & 0.22 \\
\hline & & & & Allergy & Flowers and leaves & Oral: decoction & 0.19 \\
\hline & & & & Intestinal disorders & Roots & Oral: infusion & 0.23 \\
\hline \multirow{7}{*}{ Asteraceae } & \multirow{7}{*}{$\begin{array}{l}\text { Rhaponticum acaule (L.) } \\
\text { DC. } \\
\text { (TG56) }\end{array}$} & \multirow{7}{*}{ Tafgha } & & & \multirow{3}{*}{ Roots } & Spices (Seasoning) & 0.17 \\
\hline & & & & & & Powder mixed with butter (Other: perfuming) & 013 \\
\hline & & & \multirow{5}{*}{$\mathrm{Me}$} & & & & \\
\hline & & & & Intestinal disorders & \multirow{2}{*}{ Roots } & Oral: powder & 0.12 \\
\hline & & & & $\begin{array}{l}\text { Aperitif, bronchial } \\
\text { diseases }\end{array}$ & & Oral: decoction & 0.09 \\
\hline & & & & Wound healing & Leaves, roots & Skin application: powder & 0.11 \\
\hline & & & & Hypocholesterolemic & Roots & Oral: powder & 0.15 \\
\hline \multirow[b]{2}{*}{ Asteraceae } & & & $\mathrm{F}$ & & & Condiment in tea (Other: perfuming) & 0.15 \\
\hline & $\begin{array}{l}\text { (Brot.) Vasc } \\
\text { (BJ12) }\end{array}$ & Babounj & $\mathrm{Me}$ & $\begin{array}{l}\text { sedative, digestive } \\
\text { disorders }\end{array}$ & Flowers & Oral: decoction & 0.76 \\
\hline & & & & & Tender stems & Raw (Snacks) & 0.11 \\
\hline & & & & & Young leaves, & Us ed to prepare beqoula dish (Vegetables) & 0.13 \\
\hline & Cynara humilis $\mathrm{L}$ & & $\mathrm{F}$ & & Receptacle & $\begin{array}{l}\text { Used for making domestic cheese (Other: food } \\
\text { preservation) }\end{array}$ & 0.43 \\
\hline Asteraceae & (TT23) & Timta & & & Roots & Spices (Seasoning) & 0.08 \\
\hline & & & Me & Burns & $\begin{array}{l}\text { leaves, roots, } \\
\text { stems }\end{array}$ & Skin application: powder & 0.84 \\
\hline & & & & Digestive disorders & Roots & Oral: decoction & 0.12 \\
\hline
\end{tabular}




\begin{tabular}{|c|c|c|c|c|c|c|c|}
\hline \multirow{3}{*}{ Asteraceae } & \multirow{3}{*}{$\begin{array}{l}\text { Glebionis coronaria (L.) } \\
\text { Cass. ex Spach } \\
\text { (KJ 20) }\end{array}$} & \multirow{3}{*}{$\begin{array}{l}\text { Rjel djaja, } \\
\text { kraa djaja }\end{array}$} & \multirow{3}{*}{$\mathrm{F}$} & & $\begin{array}{l}\text { Young leaves, } \\
\text { tender stems }\end{array}$ & Used to prepare beqoula dish (Vegetables) & 0.66 \\
\hline & & & & & Tender stems & Raw (Snacks) & 0.25 \\
\hline & & & & & Leaves & Cooked in the soup (Other: perfuming) & 0.15 \\
\hline \multirow{3}{*}{ Asteraceae } & \multirow{3}{*}{$\begin{array}{l}\text { Echinops spinosissimus } \\
\text { Turra } \\
\text { (TR56) }\end{array}$} & \multirow{3}{*}{ Taskra } & $\mathrm{F}$ & & Roots & Spices (Seasoning) & 0.31 \\
\hline & & & \multirow[t]{2}{*}{$\mathrm{Me}$} & $\begin{array}{l}\text { Menstrual cramps, } \\
\text { cold, fractures }\end{array}$ & Whole plant & Oral: decoction & 0.18 \\
\hline & & & & Anemia & Roots & Oral: powder & 0.07 \\
\hline \multirow{4}{*}{ Asteraceae } & \multirow{4}{*}{$\begin{array}{l}\text { Scolymus hispanicus L } \\
\text { (GR1) }\end{array}$} & \multirow{4}{*}{ El guernina } & \multirow{3}{*}{$\mathrm{F}$} & & $\begin{array}{l}\text { Young leaves, } \\
\text { tender stems }\end{array}$ & $\begin{array}{l}\text { Prepared like spinach (Vegetables) } \\
\text { Used to prepare begoula dish (Vegetables) }\end{array}$ & $\begin{array}{ll}0.26 \\
0.22\end{array}$ \\
\hline & & & & & $\begin{array}{l}\text { Roots, tender } \\
\text { stems }\end{array}$ & Cooked in broth with meat (Vegetables) & 0.80 \\
\hline & & & & & Tender stems & Used to prepare salad (Vegetables) & 0.17 \\
\hline & & & $\mathrm{Me}$ & Cough, cold & Roots & Inhalation: fumigation & 0.07 \\
\hline Asteraceae & $\begin{array}{l}\text { Silybum marianum }(\mathrm{L} .) \\
\text { Gaertn } \\
\text { (CH27) }\end{array}$ & Choka hmar & $\mathrm{F}$ & & Receptacle & Raw (Snacks) & 0.29 \\
\hline \multirow{5}{*}{ Brassicaceae } & \multirow{5}{*}{$\begin{array}{l}\text { Diplotaxis catholica (L.) DC } \\
\text { (HR 62) }\end{array}$} & \multirow{5}{*}{ Kalkaza } & $\mathrm{F}$ & & $\begin{array}{l}\text { Young leaves, } \\
\text { tender stems }\end{array}$ & Used to prepare beqoula dish (Vegetables) & 0.23 \\
\hline & & & \multirow{4}{*}{$\mathrm{Me}$} & Ear diseases & Whole plant & Pressed and used as ear drops & 0.22 \\
\hline & & & & Anemia & Leaves, stems & Oral: infusion & 0.17 \\
\hline & & & & Hemorrhoids & Whole plant & Skin application: poultice & 0.09 \\
\hline & & & & Asthma & Flowers & Oral: powder & 0.19 \\
\hline \multirow[t]{2}{*}{ Brassicaceae } & \multirow{2}{*}{$\begin{array}{l}\text { Raphanus raphanistrum } \mathrm{L} \\
\text { (BH12) }\end{array}$} & \multirow{2}{*}{$\begin{array}{l}\text { Bouhammo } \\
u\end{array}$} & $\mathrm{~F}$ & & $\begin{array}{l}\text { Leaves, tender } \\
\text { stems }\end{array}$ & Used to prepare beqoula dish (Vegetables) & 0.28 \\
\hline & & & $\mathrm{Me}$ & Shortness of breath & Leaves & Oral: infusion & 0.12 \\
\hline Brassicaceae & $\begin{array}{l}\text { Sinapis alba L } \\
\text { (HA12) }\end{array}$ & $\begin{array}{l}\text { Elharra, el- } \\
\text { laftiya }\end{array}$ & $\mathrm{F}$ & & $\begin{array}{l}\text { Tender stems, } \\
\text { leaves }\end{array}$ & Used to prepare beqoula dish (Vegetables) & 0.10 \\
\hline \multirow[t]{2}{*}{ Caryophyllaceae } & \multirow{2}{*}{$\begin{array}{l}\text { Herniaria hirsuta subsp. } \\
\text { cinerea (DC.) Cout. } \\
\text { (HL63) }\end{array}$} & \multirow{2}{*}{$\begin{array}{l}\text { Harassat } \\
\text { lehjar }\end{array}$} & $\mathrm{F}$ & & & & \\
\hline & & & $\mathrm{Me}$ & $\begin{array}{l}\text { Kidney stones, } \\
\text { Intestinal disorders }\end{array}$ & Leaves & Oral: decoction & 0.81 \\
\hline \multirow{2}{*}{ Caryophyllaceae } & \multirow{2}{*}{$\begin{array}{l}\text { Paronychia argentea Lam } \\
\text { (MF37) }\end{array}$} & \multirow{2}{*}{ Mosuif } & $\mathrm{F}$ & & Leaves & Spices (Seasoning) & 0.34 \\
\hline & & & $\mathrm{Me}$ & Wound healing & Leaves & Skin application: powder & 0.09 \\
\hline \multirow{3}{*}{ Caryophyllaceae } & \multirow{3}{*}{$\begin{array}{l}\text { Silene vulgaris (Moench) } \\
\text { Garcke } \\
\text { (TC39) }\end{array}$} & \multirow{3}{*}{ Tighecht } & $\mathrm{F}$ & & Leaves & Used to prepare beqoula dish (Vegetables) & 0.09 \\
\hline & & & $\mathrm{Me}$ & $\begin{array}{l}\text { To rid the body of } \\
\text { toxins }\end{array}$ & Whole plant & Oral: decoction & 0.26 \\
\hline & & & Other & & Roots & Washing of clothing, wool and the laundry & 0.89 \\
\hline
\end{tabular}


Ethnobotany Research and Applications

\begin{tabular}{|c|c|c|c|c|c|c|c|}
\hline \multirow{3}{*}{ Cyperaceae } & \multirow{3}{*}{$\begin{array}{l}\text { Cyperus rotundus } \mathrm{L} \\
\text { (TM56) }\end{array}$} & \multirow{3}{*}{ Tamousayt } & \multicolumn{2}{|l|}{$\mathrm{F}$} & \multirow{3}{*}{ Roots } & Spices (Seasoning) & 0.13 \\
\hline & & & \multirow{2}{*}{$\mathrm{Me}$} & Strengthen the hair & & External application: poultice & 0.66 \\
\hline & & & & Allergy & & Oral: powder. & 0.09 \\
\hline \multirow{4}{*}{ Euphorbiaceae } & \multirow{4}{*}{$\begin{array}{l}\text { Mercurialis annua L } \\
\text { (HS3) }\end{array}$} & \multirow{4}{*}{$\begin{array}{l}\text { Horriga } \\
\text { Imalssa }\end{array}$} & \multirow[b]{2}{*}{$\mathrm{F}$} & & \multirow[b]{2}{*}{ Young leaves, } & Used to prepare beqoula dish (Vegetables) & 0.20 \\
\hline & & & & & & $\begin{array}{l}\text { Powder mixed with the barley semolina to prepare } \\
\text { the couscous (Other: perfuming) }\end{array}$ & 0.15 \\
\hline & & & & Cold, Rheumatism & Leaves & Oral: cooked in the soup & 0.11 \\
\hline & & & $\mathrm{Me}$ & $\begin{array}{l}\text { Intestinal Disorders, } \\
\text { Kidney Disease, } \\
\text { Dysuria Problems } \\
\end{array}$ & Leaves & Oral: decoction & 0.16 \\
\hline Fabaceae & $\begin{array}{l}\text { Astragalus boeticus L } \\
\text { (KB40) }\end{array}$ & Krenbouch & $\mathrm{F}$ & & Seeds & Raw (Snacks) & 0.93 \\
\hline Fabaceae & $\begin{array}{l}\text { Lathyrus clymenum L } \\
\text { (RN40) }\end{array}$ & Rig elhench & $\mathrm{F}$ & & Seeds & Raw (Snacks) & 0.36 \\
\hline \multirow[t]{2}{*}{ Fabaceae } & \multirow{2}{*}{$\begin{array}{l}\text { Scorpiurus muricatus L } \\
\text { (KW37) }\end{array}$} & \multirow{2}{*}{$\begin{array}{l}\text { El-kelwa, } \\
\text { kelwat rnab }\end{array}$} & \multirow[t]{2}{*}{$\mathrm{F}$} & & $\begin{array}{l}\text { Young leaves, } \\
\text { stems }\end{array}$ & Used to prepare beqoula dish (Vegetables) & 0.31 \\
\hline & & & & & Tender stems & Raw (Snacks) & 0.11 \\
\hline \multirow{2}{*}{ Iridaceae } & \multirow{2}{*}{$\begin{array}{l}\text { Moraea sisyrinchium (L.) } \\
\text { Ker Gawl } \\
\text { (SW43) }\end{array}$} & \multirow[t]{2}{*}{ Lmessiw } & $\mathrm{F}$ & & Rhizome & Raw (Snacks) & 0.32 \\
\hline & & & $\mathrm{Me}$ & Caugh & Roots & Oral: decoction & 0.06 \\
\hline \multirow{4}{*}{ Juncaceae } & \multirow{4}{*}{$\begin{array}{l}\text { Juncus acutus L } \\
\text { (SE60) }\end{array}$} & \multirow{4}{*}{ Essmar } & $\mathrm{F}$ & & & Condiment in soup (Other: perfuming) & 0.10 \\
\hline & & & \multirow[b]{2}{*}{$\mathrm{Me}$} & Rheumatism & Seeds & Oral: raw & 0.08 \\
\hline & & & & Kidney stones & Leaves & Oral: infusion & 0.17 \\
\hline & & & Other & & Stems & making traditional dishes, rugs, and brooms & 0.87 \\
\hline \multirow{4}{*}{ Lamiaceae } & \multirow{4}{*}{$\begin{array}{l}\text { Ajuga iva (L.) Schreb. } \\
\text { (CG5) }\end{array}$} & \multirow{4}{*}{$\begin{array}{l}\text { Chendgour } \\
\text { a }\end{array}$} & \multirow{3}{*}{$\mathrm{F}$} & & \multirow{3}{*}{ Young leaves } & $\begin{array}{l}\text { Powder mixed with the bread flour (Other: } \\
\text { perfuming) }\end{array}$ & 0.69 \\
\hline & & & & & & Used to prepare beqoula dish (Vegetables) & 0.27 \\
\hline & & & & & & Spices (Seasoning) & 0.42 \\
\hline & & & $\mathrm{Me}$ & Abdominal pain, cold & Leaves & Oral: decoction & 0.19 \\
\hline \multirow{2}{*}{ Lamiaceae } & \multirow{2}{*}{$\begin{array}{l}\text { Lavandula multifida L } \\
\text { (ZR22) }\end{array}$} & \multirow{3}{*}{$\begin{array}{l}\text { Zririka, } \\
\text { kohhila }\end{array}$} & $\mathrm{F}$ & & Leaves & Tisane (Other: perfuming) & 0.24 \\
\hline & & & $\mathrm{Me}$ & Caugh, cold & Whole plant & Oral: infusion & 0.36 \\
\hline \multirow[b]{3}{*}{ Lamiaceae } & & & $\mathrm{F}$ & & & Spices (Seasoning) & 0.04 \\
\hline & & & & Digestive disorders & Roots & Oral: powder & 0.25 \\
\hline & $\begin{array}{l}\text { Salvia verbenaca } \mathrm{L} \\
\text { (KW50) }\end{array}$ & $\begin{array}{l}\text { Khwiwita, } \\
\text { khiyata }\end{array}$ & $\mathrm{Me}$ & $\begin{array}{l}\text { Wound healing, skin } \\
\text { diseases }\end{array}$ & Whole plant & Skin application: powder & 0.75 \\
\hline & & & $\mathrm{F}$ & & Leaves & Cooked with the snail (Other: perfuming) & 0.33 \\
\hline Lamiaceae & $\begin{array}{l}\text { Thymus broussonetul Boiss } \\
\text { (ZH30) }\end{array}$ & $\begin{array}{l}\text { Zaatar } \\
\text { lhmir }\end{array}$ & $\mathrm{Me}$ & $\begin{array}{l}\text { Digestive disorders, } \\
\text { cold }\end{array}$ & Aerial parts & Oral: decoction & 0.25 \\
\hline
\end{tabular}




\begin{tabular}{|c|c|c|c|c|c|c|c|}
\hline Malvaceae & $\begin{array}{l}\text { Malva multiflora (Cav.) } \\
\text { Soldano, Banfi \& Galasso } \\
\text { (KZ1) }\end{array}$ & $\begin{array}{l}\text { El- } \\
\text { khobbiza, } \\
\text { El-bakkoula }\end{array}$ & $\mathrm{F}$ & & $\begin{array}{l}\text { Young leaves, } \\
\text { tender stems }\end{array}$ & Used to prepare Beqoula dish (Vegetables) & 0.99 \\
\hline \multirow{8}{*}{ Molluginaceae } & \multirow{8}{*}{$\begin{array}{l}\text { Corrigiola littoralis subsp. } \\
\text { telephiifolia (Pourr.) Briq } \\
\text { (SG28) }\end{array}$} & \multirow{8}{*}{ Serghina } & \multirow{3}{*}{\multicolumn{2}{|c|}{$\mathrm{F}$}} & Roots & Powder mixed with bread flour (Other: perfuming) & 0.34 \\
\hline & & & & & Tender stems & Used to prepare beqoula dish (Vegetables) & 0.08 \\
\hline & & & & & Leaves & Spices (Seasoning) & 0.27 \\
\hline & & & \multirow{4}{*}{$\mathrm{Me}$} & Allergy & \multirow{3}{*}{ Roots } & Oral: powder & 0.28 \\
\hline & & & & To prevent hair loss & & Externe application: poultice & 0.11 \\
\hline & & & & $\begin{array}{l}\text { To rid the body of } \\
\text { toxins (tokal) }\end{array}$ & & Oral: infusion & 0.12 \\
\hline & & & & Cold, diarrhea & Whole plant & Oral: decoction & 0.05 \\
\hline & & & Other & & Roots & Encens & 0.28 \\
\hline \multirow[t]{2}{*}{ Oxalidaceae } & \multirow[t]{2}{*}{$\begin{array}{l}\text { Oxalis pes-caprae L } \\
\text { (MD10) }\end{array}$} & \multirow[t]{2}{*}{$\begin{array}{l}\text { Elhomida } \\
\text { safra }\end{array}$} & \multirow[t]{2}{*}{$\mathrm{F}$} & & Young leaves & Used to prepare beqoula dish (Vegetables) & 0.36 \\
\hline & & & & & Tender stems & Raw (Snacks) & 0.75 \\
\hline \multirow{4}{*}{ Palmaceae } & \multirow{4}{*}{$\begin{array}{l}\text { Chamaerops humilis } \mathrm{L} \\
\text { (DD53) }\end{array}$} & \multirow{4}{*}{$\begin{array}{l}\text { Eddoum, } \\
\text { lghaz }\end{array}$} & $\mathrm{F}$ & & & Raw (Snacks) & 0.21 \\
\hline & & & \multirow[t]{2}{*}{ Me } & \multirow[t]{2}{*}{ Digestive disorders } & Fruits & Oral: Raw & 0.44 \\
\hline & & & & & Roots & Oral: powder & 0.13 \\
\hline & & & Other & & Leaves & Manufacture of brooms, baskets and traditional hats & 0.89 \\
\hline \multirow{5}{*}{ Papaveraceae } & \multirow{5}{*}{$\begin{array}{l}\text { Papaver rhoeas } \mathrm{L} \\
\text { (LL12) }\end{array}$} & \multirow{5}{*}{ Belaaman } & $\mathrm{F}$ & & Young leaves, & Used to prepare beqoula dish (Vegetables) & 0.84 \\
\hline & & & \multirow{4}{*}{$\mathrm{Me}$} & Measles fever & Flowers & Oral: infusion & 0.16 \\
\hline & & & & Meastes, tever & \multirow{2}{*}{ Leaves } & Skin application: poultice & 0.24 \\
\hline & & & & Sedative & & Oral: infusion & 0.14 \\
\hline & & & & Hypocholesterolemic & Flowers & Oral: infusion & 0.13 \\
\hline \multirow{3}{*}{ Papaveraceae } & \multirow{3}{*}{$\begin{array}{l}\text { Fumaria agraria Lag } \\
\text { (EB39) }\end{array}$} & \multirow{3}{*}{$\begin{array}{l}\text { Ennar } \\
\text { elbarda }\end{array}$} & $\mathrm{F}$ & & \multirow{3}{*}{ Leaves } & Used to prepare beqoula dish (Vegetables) & 0.04 \\
\hline & & & Mo & Wounds, Antipruritic & & Skin application: extract, powder & 0.47 \\
\hline & & & Me & Fever & & Oral: infusion & 0.14 \\
\hline \multirow{2}{*}{ Plumbaginaceae } & \multirow{2}{*}{$\begin{array}{l}\text { Limonium sinuatum (L.) } \\
\text { Mill } \\
\text { (KT35) }\end{array}$} & \multirow{2}{*}{$\begin{array}{l}\text { Khnounet } \\
\text { ennaja }\end{array}$} & $\mathrm{F}$ & & Tender leaves & Raw (Snacks) & 0.07 \\
\hline & & & $\mathrm{Me}$ & Skin diseases & Leaves & Skin application: poultice & 0.19 \\
\hline \multirow{2}{*}{ Poaceae } & \multirow{2}{*}{$\begin{array}{l}\text { Cynodon dactylon (L.) Pers } \\
\text { (EN35) }\end{array}$} & \multirow{2}{*}{ Enjem } & $\mathrm{F}$ & & \multirow{2}{*}{ Roots } & Spices (Seasoning) & 0.23 \\
\hline & & & $\mathrm{Me}$ & Rheumatism, cold & & Oral: decoction & 0.31 \\
\hline Poaceae & $\begin{array}{l}\text { Lolium multiflorum Lam } \\
\text { (MN67) }\end{array}$ & $\begin{array}{l}\text { El- } \\
\text { medhoun }\end{array}$ & $\mathrm{F}$ & & Roots & Spices (Seasoning) & 0.09 \\
\hline
\end{tabular}




\section{Ethnobotany Research and Applications}

\begin{tabular}{|c|c|c|c|c|c|c|c|}
\hline \multirow{3}{*}{ Polygonaceae } & \multirow{3}{*}{$\begin{array}{l}\text { Rumex pulcher } \mathrm{L} \\
(\mathrm{HH} 12)\end{array}$} & \multirow{3}{*}{$\begin{array}{l}\text { Hommaida } \\
\text { mchowka }\end{array}$} & \multicolumn{2}{|l|}{$\mathrm{F}$} & \multirow{3}{*}{$\begin{array}{l}\begin{array}{l}\text { Young leaves, } \\
\text { tender stems }\end{array} \\
\text { Leaves }\end{array}$} & \multirow{2}{*}{$\begin{array}{l}\text { Used to prepare beqoula dish (Vegetables) } \\
\text { Oral: infusion }\end{array}$} & \multirow{2}{*}{$\begin{array}{l}0.17 \\
0.11 \\
\end{array}$} \\
\hline & & & $\mathrm{Me}$ & Cold & & & \\
\hline & & & Othe & & & rubbing copper utensils & 0.36 \\
\hline \multirow{2}{*}{ Polygonaceae } & \multirow{2}{*}{$\begin{array}{l}\text { Emex spinosa (L.) Campd } \\
\text { (HS5) }\end{array}$} & \multirow{2}{*}{$\begin{array}{l}\text { Hommaida } \\
\text { hamrat-rass }\end{array}$} & $\mathrm{F}$ & & Tender stems & Raw (Snacks) & 0.15 \\
\hline & & & $\mathrm{Me}$ & Digestive disorders & Fruits, leaves & Oral: infusion & 0.09 \\
\hline Portulacaceae & $\begin{array}{l}\text { Portulaca oleracea L } \\
\text { (ER5) }\end{array}$ & Errejla & $\mathrm{F}$ & & $\begin{array}{l}\text { Young leaves, } \\
\text { tender stems }\end{array}$ & Used to prepare beqoula dish (Vegetables) & 0.95 \\
\hline \multirow[b]{2}{*}{ Rubiaceae } & \multirow{2}{*}{$\begin{array}{l}\text { Rubia peregrina L } \\
\text { (WW12) }\end{array}$} & \multirow[b]{2}{*}{ El fouwwa } & $\mathrm{F}$ & & \multirow[b]{2}{*}{ Roots } & Spices (Seasoning) & 0.35 \\
\hline & & & $\mathrm{Me}$ & Anemia & & Oral: decoction, powder & 0.79 \\
\hline \multirow[b]{2}{*}{ Scrophulariaceae } & \multirow[b]{2}{*}{$\begin{array}{l}\text { Verbascum sinuatum } \mathrm{L} \\
\text { (SH9) }\end{array}$} & \multirow[b]{2}{*}{ maslahndar } & $\mathrm{F}$ & & Leaves & Spices (Seasoning) & 0.09 \\
\hline & & & $\mathrm{Me}$ & Cataract & Flowers & $\begin{array}{l}\text { External application: the water that accumulates in } \\
\text { the Flowers is used as eye drops }\end{array}$ & 0.12 \\
\hline \multirow{3}{*}{ Solanaceae } & \multirow{3}{*}{$\begin{array}{l}\text { Solanum americanum Mill } \\
\text { (DD76) }\end{array}$} & \multirow{3}{*}{ Eneb eddib } & $\mathrm{F}$ & & Fruits & Raw (Snacks) & 0.11 \\
\hline & & & \multirow[b]{2}{*}{$\mathrm{Me}$} & Fever & Leaves & Skin application: decoction & 0.07 \\
\hline & & & & $\begin{array}{l}\text { Hypoglycaemic, } \\
\text { digestive disorders }\end{array}$ & Fruits leaves & Oral: cooked & 0.08 \\
\hline \multirow{3}{*}{ Urticaceae } & \multirow{3}{*}{$\begin{array}{l}\text { Urtica dioica L } \\
\text { (HH12) }\end{array}$} & \multirow{3}{*}{$\begin{array}{l}\text { Horiga } \\
\text { harcha }\end{array}$} & $\mathrm{F}$ & & \multirow{3}{*}{ Leaves } & Cooked in the soup (Other: perfuming) & 0.07 \\
\hline & & & \multirow[b]{2}{*}{$\mathrm{Me}$} & Rheumatism & & Skin application: rubbing on the skin & 0.70 \\
\hline & & & & $\begin{array}{l}\text { Cold, anemia, canker } \\
\text { sores }\end{array}$ & & Oral: infusion & 0.22 \\
\hline
\end{tabular}

F= Food, Me= Medicinal 
For wild plants of medicinal use, Dysphania ambrosioides (L.) Mosyakin \& Clemants (RFC =0.93), Cynara humilis L. (RFC $=0.84)$, Herniaria hirsuta (RFC $=0.81)$, Rubia peregrina L. (RFC $=0.79)$, Chamaemelum fuscatum (Brot.) Vasc. $(\mathrm{RFC}=0.76)$ and Salvia verbenaca $\mathrm{L} .(\mathrm{RFC}=0.75)$ were the most cited plants in the study area. These results are different from those of the many studies that have cited species such as Artemisia herba-alba Asso and Mentha pulegium L. as highly recommended plants in various regions of Morocco (Barkaoui et al. 2017, Eddouks et al. 2017, Fakchich \& Elachouri 2014, Teixidor-Toneu et al. 2016).

Wild plants for medicinal use: used part, preparation and administration methods, and the various therapeutic uses

For plants for medicinal use in the study region, leaves were the most used parts (35\%). Similar results have been found in other regions of Morocco (Chaachouay et al. 2019, Eddouks et al. 2017, Teixidor-Toneu et al. 2016). These results were explained by the central role of the leaves for biochemical reactions, including photosynthesis (Salhi et al. 2019). Other parts of the plants were also used: roots (25\%), stems (11\%), flowers (8\%), seeds (4\%) and other parts (17\%) (Fig. 3).

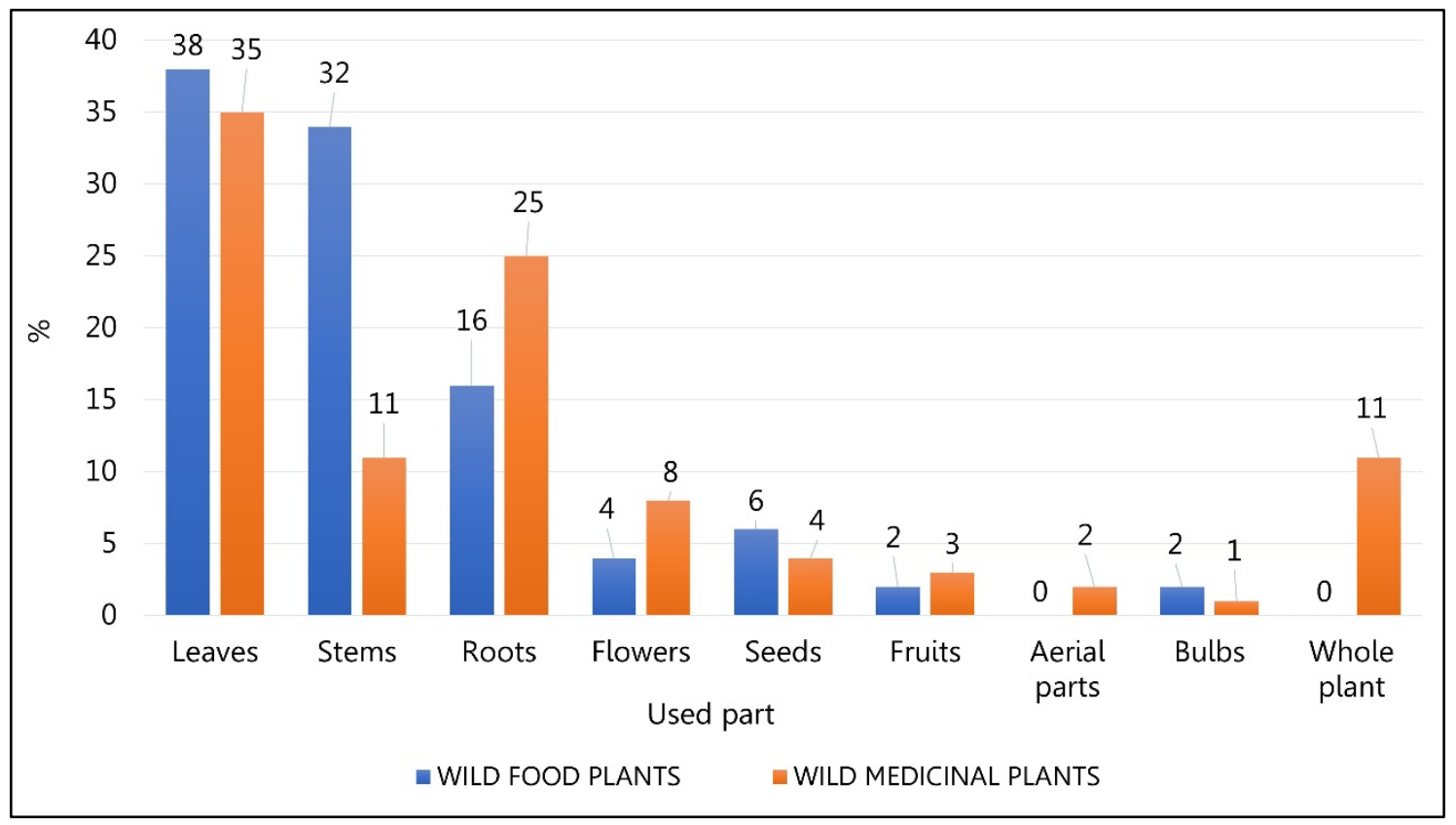

Figure 3. Distribution of the plant parts used.

Decoction was the most frequent method of preparation (27.27\%) followed by drying and consumption in powder form (22.73\%) and finally the infusion (19.32\%). Other less common preparation methods (poultice, raw, cooked, etc.) combine to make up $30.68 \%$ of the total. The appreciation of the decoction as a method of preparation has been mentioned in other ethnobotanical studies carried out in Morocco (Barkaoui et al. 2017, El Abbouyi et al. 2014, Mrabti et al. 2019, Redouan et al. 2020).

The majority of preparations were administered orally (80.25\%) followed by skin applications (13.58\%) and external applications (2.47\%). The large proportion of oral administration of wild plants in the present study is in agreement with some other research carried out in the country (Chaachouay et al. 2019, El Abbouyi et al. 2014, Mrabti et al. 2019).

\section{Wild plants used for food purposes: used part and methods of preparation}

Analysis of data for wild plants used as food showed that the leaves (38\%) and stems (34\%) were the parts most used by the local population. The widespread use of the leaves could be attributed to the abundance and easy access to this organ compared to other organs (Neves et al. 2009), similarly the latter were a key determinant in differentiating and identifying these plants (Neves et al. 2009). These results also show that the aerial parts of plants play an important role in the culinary preparation of plants for food use in the province of Sidi Bennour. Roots are the third most used plant organ (16\%) in the study area, after leaves and stems. They are used as a spice in several preparations.

Four categories of plant use were mentioned. They were used as vegetables, for seasoning, as a beverage and for other uses (which includes plants used to decorate or flavor traditional dishes) (Table 2). Vegetables (37.5\%) represent the most common mode of consumption of WEPs. They use these species to prepare traditional dishes such as broth or couscous. However, the majority of WEPs are used to prepare the beqoula dish, considered to be 
the most common method of consuming WEPs in rural Morocco (Nassif \& Tanji 2013). This dish is prepared with the Malva multiflora plant alone (Fig. 4), with Portulaca oleracea L alone, or prepared by a combination of several plants (Fig. 5) depending on the preferred taste. The preparation of this dish uses plants collected at the young stage from uncultivated land, cut and cooked with water or steam after washing, and adds spices such as ginger, pepper and paprika. Other ingredients such as parsley, olive oil, olives, lemon, chilli, and salt are also added to season the dish and improve its taste. The use of the beqoula dish by the Moroccan population was mentioned by many other studies (Nassif \& Tanji 2013, Powell et al. 2014, Tbatou et al. 2016a, Tbatou et al. 2016b).

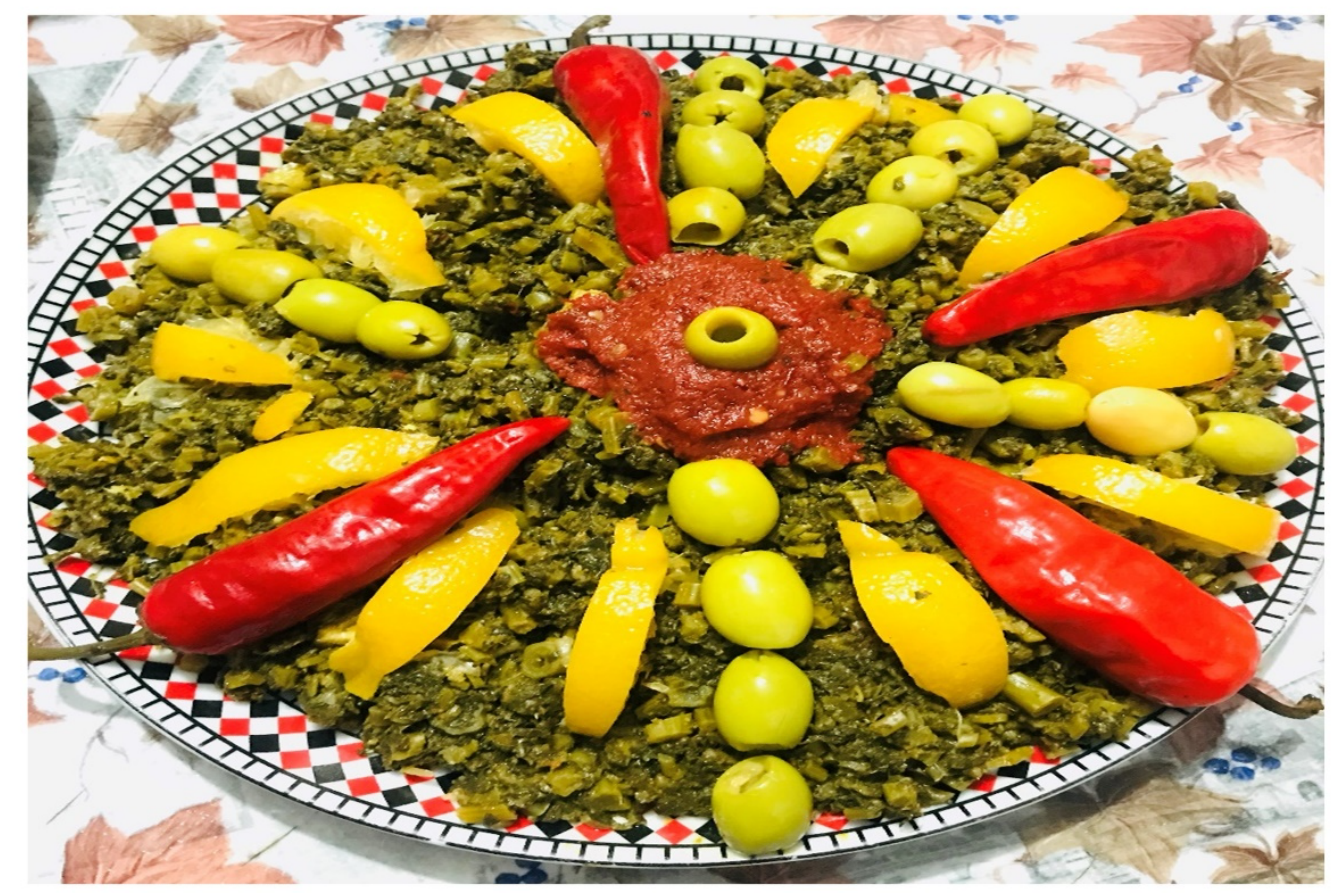

Figure 4. beqoula dish

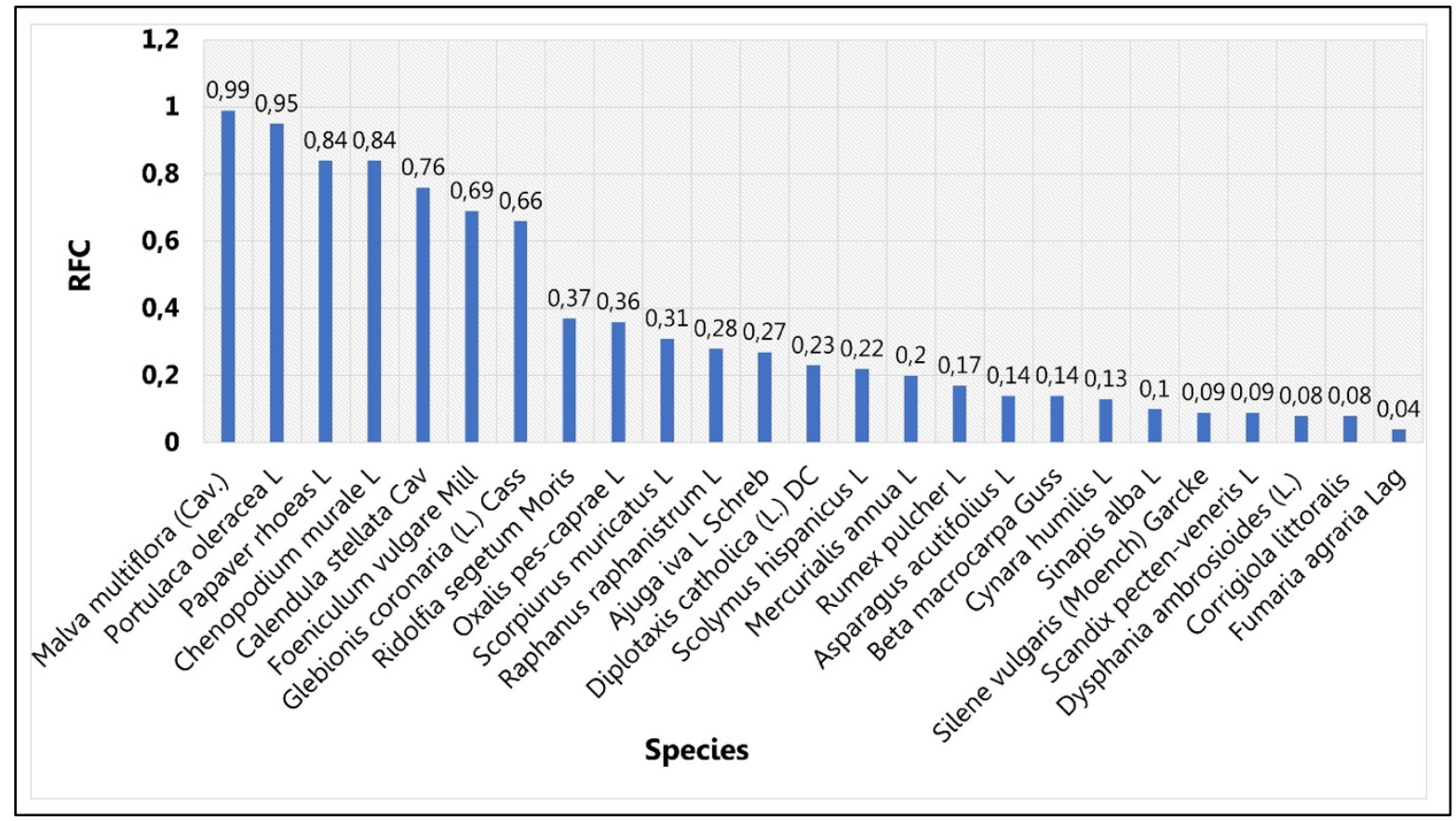

Figure 5. plants used in the preparation of the beqoula dish according to RFC in the region of Sidi Bennour

In addition to the food and medicinal use of WEPs, other types of uses have been reported by our study. This is the case of Silene vulgaris (Moench) Garcke, formerly used to wash clothes and mattresses as cleaning products, Rumex pulcher L., that was also used to scrub copper utensils, Chamaerops humilis L. and Juncus acutus L. have been 
exploited to make local products such as brooms, baskets, traditional dishes, and traditional hats (Fig. 6) which will be sold in markets and souks to provide income.

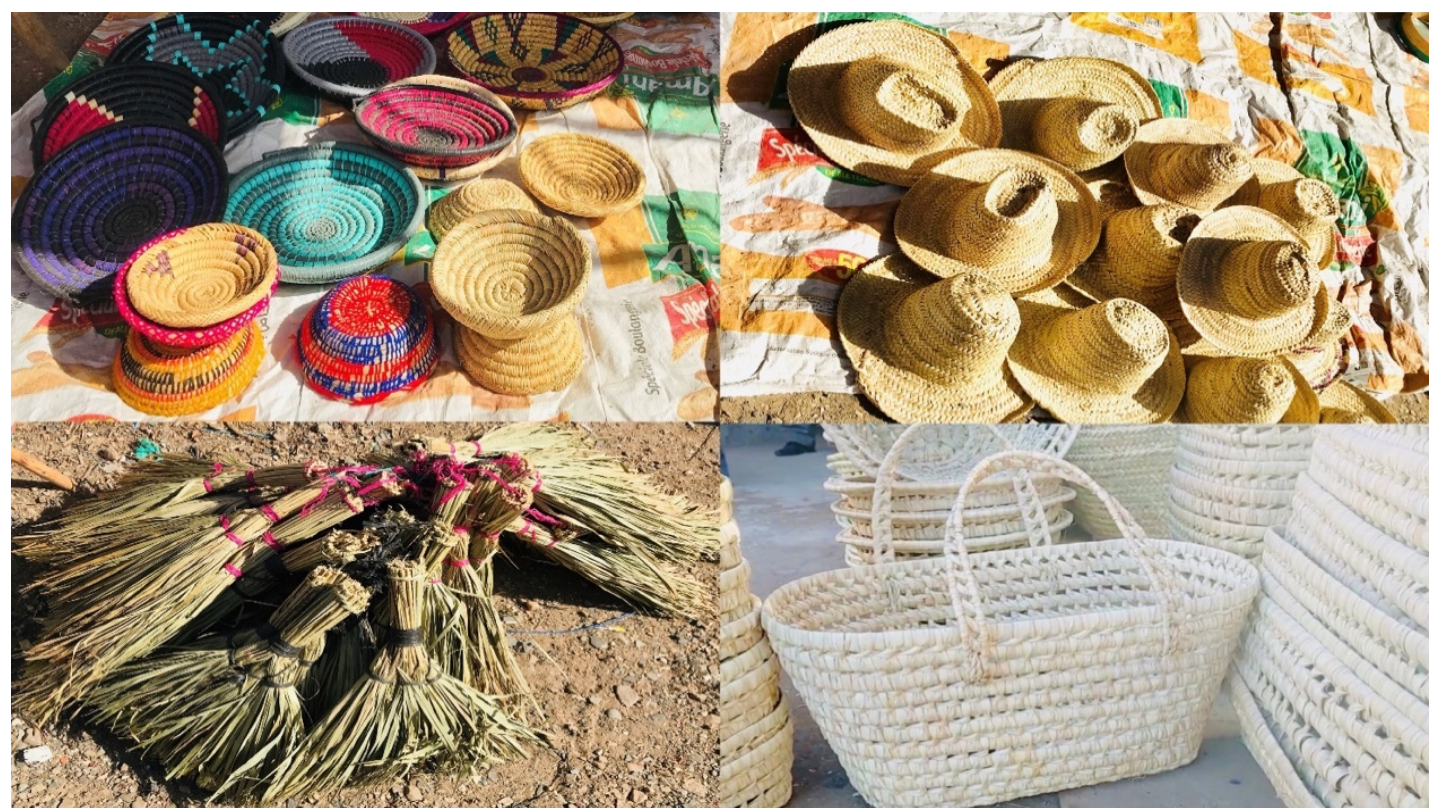

Figure 6. local products made from the leaves of the species Chamaerops humilis for sale in the souk of Sidi Bennour

The use of wild plants as a seasoning or spice is also appreciated by the people of this region. Several plants are combined to prepare some seasonings. The major organ for preparing them is the root, but other organs can be used, for example the leaves of the species Ajuga iva L. Schreb, the flowers of Calendula stellata Cav and the fruits of Foeniculum vulgare Mill. These spices are added to a range of traditional dishes used in winter as a means of warming the body, hence their local name "Msakhen". WEPs are also eaten raw as a snack, by the population of the region and more particularly, by shepherds when they are in the field, children when they go to school and women when they collect these plants in the field. The most consumed part in this mode are the young stems after removing the outer layers. Such use has been mentioned in other research (Nassif \& Tanji 2013, Tbatou et al. 2016a, Tbatou et al. 2016b).

The listed wild medicinal plants have been used by locals for the treatment of a number of common diseases and disorders. Forty-two disease types belonging to 13 different pathological groups have been listed. Indeed, the local population declared to use the medicinal plants against dermatological affections, gastrointestinal disorders, respiratory system diseases, rheumatic diseases, hepatic, and metabolic problems, uro-genital disorders, eye diseases, appetite stimulants, antiseptic, febrifuge, haircare, analgesic, and infectious diseases. Most of these plants had more than one therapeutic use. For example, Allium roseum $\mathrm{L}$ is used to treat jaundice, colds, rheumatism, and stomach upset. The most cited pathologies as shown in Fig. 7 were respiratory system diseases (20.18\%), gastrointestinal disorders (18.42\%) and hepatic and metabolic problems (17.54\%)

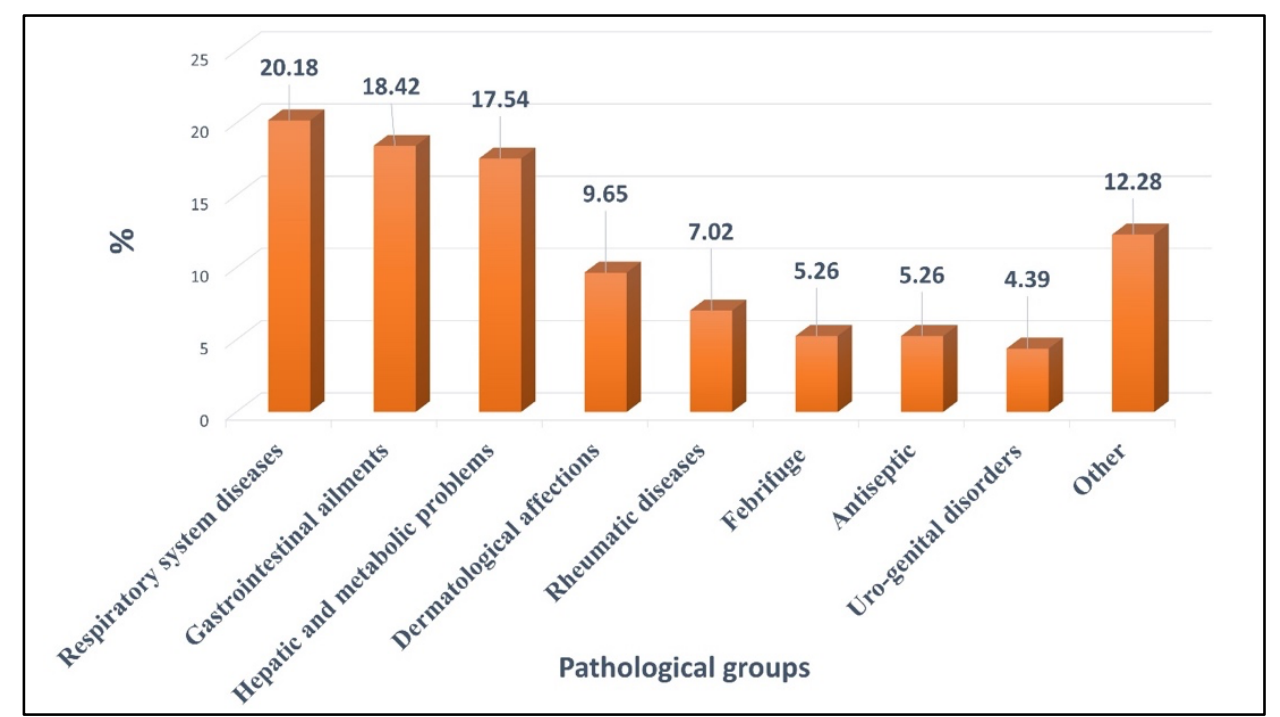

Figure 7. Reported ailment groups. 
Safe use of wild edible plants

Despite the low formal educational attainment of the rural population compared to the urban population, the former, especially women and the elderly in rural areas, have sufficient information to allow them to use WEPs safely. An example is that Arisarum vulgare. O. Targ-Tozz (Yarni) was reported by the study population to be a poisonous plant, but widely used during periods of famine as food. Specific preparation methods to remove this toxicity have been adopted by the population by boiling the bulbs for a long time ( $24 \mathrm{hrs}$ ) or repeatedly ( 7 times). Previous studies have shown that the toxicity of this plant is due to the presence of alkaloids and mainly a major alkaloid, irniine (Melhaoui et al. 1992). Another toxic plant Citrullus colocynthis (L.) Schrad., mentioned by the population of Sidi Bennour, has also been reported in several experiments and previous studies (Al-Yahya et al. 2000; Bakhiet \& Adam 1995; Diwan et al. 2000). This medicinal plant is used by the local population to treat diabetes, and to avoid its toxicity, precise quantities of seeds (not exceeding 5 ) are used by the population.

\section{Exploitation trend of wild edible plants}

According to the population surveyed, there is a sharp decline in the consumption of most of the species recorded, whether for food or medicinal use, especially among the younger generation. Several reasons were put forward to explain, at least in part, this phenomenon. According to several interviewees, the use of these plants has decreased because their consumption is no longer necessary, given the availability of cultivated food plants and pharmaceuticals considered to be more effective and with a rapid effect compared to wild plants. In addition, the availability of these plants has decreased due to climate change and overuse of herbicides as these plants are considered weeds. These results corroborate those of Luczaj et al (2012), Broegaard et al (2017), and Ickowitz et al (2019), who suggest that under conditions where food resources are abundant human populations tend to prefer industrialized products. This thus reduces the dietary diversity of these populations, while under conditions of scarcity of resources; they used these wild plants to meet their nutritional and therapeutic needs. Caniago \& Stephen (1998) have shown that the increasing use of pharmaceuticals is a fundamental determinant of the decrease in the exploitation of medicinal plants among indigenous populations. However, this decrease does not affect all plants with the same intensity as many WEPs are still widely used today, such as Malva multiflora (Cav.) Soldano, Banfi \& Galasso, Portulaca oleracea L, Astragalus boeticus L, Dysphania ambrosioides (L.) Mosyakin \& Clemants, Herniaria hirsuta subsp. cinerea (DC.) Cout, Rubia peregrina L, Ridolfia segetum(L.) Moris, Chamaemelum fusccatum and Cynara humilis.

\section{Conclusion}

The Sidi Bennour region is home to an abundant diversity of wild plants with 56 species documented by this study, which have medicinal uses, food uses, or both. The people of this region, especially the elderly women, hold a rich traditional knowledge of a large number of WEPs. They have sufficient information to allow them to use these plants safely. However, this traditional knowledge and know-how is threatened due to the lack of successors. Indeed, there is a lack of interest in these plants among the younger generations who are more drawn to the modern way of life and modern culture. In addition, the populations of many species commonly used as food or medicine is declining in the study area.

Further research on WEPs in other regions of Morocco is recommended to develop methods and decide on strategies for the conservation of these WEPs and the transmission of traditional ethnobotanical knowledge relating to their uses. There is also a need to revitalize traditional practices related to the use of these plants to regain the interest of the younger generation. In addition, it is strongly recommended to determine the nutritional potential, pharmacological and toxic effects of these plants which could offer an alternative for nutritional deficiencies and food insecurity.

\section{Declarations}

List of abbreviations: WEPs = wild edible plants, RFC= Relative Citation Frequency Index

Ethics approval and consent to participate: Consent was sought from all participants before any interviews were conducted. Tthey were also informed about the framework in which the work is carried out.

Consent for publication: Not applicable.

Data Availability: Data used to support the findings of this study are available from the corresponding author upon request.

Competing interests: Author declares that there is no conflict of interest.

Funding: This study received no funding.

However, the study benefited from the annual allocation granted by the Ministry of Higher Education and

Research (Morocco) for the functioning of Research Structures

Authors' contributions: $A A, K N, S M, K S$ : Study design, ethnobotany surveys conduction, plant collection, $A A, M T$, AK: data analysis and interpretation, manuscript writing, JMR, RB: Work supervising, contribution to Methodology, manuscript improving, and Review-Editing. All authors read, reviewed, and approved thefinal version of the paper. 


\section{Acknowledgements}

We would like to thank all the informants who contributed to this study with their valuable traditional knowledge. The study was supported by the Moroccan Ministry of Higher Education and Research. This work is also in line with the work from COST Action 18101 SOURDOMICS-Sourdough biotechnology network towards novel, healthier and sustainable food and bioprocesses (https://sourdomics.com/; https://www.cost.eu/actions/CA18101/, where the author J.M.F.R. is the Chair and Grant Holder Scientific Representative, and the author R.B. is member of Morocco as COST Near Neighbor Country.

\section{Literature cited}

Aboukhalaf A, El Amraoui B, Tabatou M, da Rocha JMF, Belahsen R. 2020. Screening of the antimicrobial activity of some extracts of edible wild plants in Morocco. Functional Foods in Health and Disease 10:265-273.

Al-Yahya M, Al-Farhan A, Adam S. 2000. Preliminary toxicity study on the individual and combined effects of Citrullus colocynthis and Nerium oleander in rats. Fitoterapia 71:385-391.

Ali-Shtayeh MS, Jamous RM, Al-Shafie JH, Wafa'A E, Kherfan FA, Qarariah KH, Isra'S K, Soos IM, Musleh AA, Isa BA. 2008. Traditional knowledge of wild edible plants used in Palestine (Northern West Bank): a comparative study. Journal of Ethnobiology and Ethnomedicine 4: 13.

Ayantunde AA, Briejer M, Hiernaux P, Udo HM, Tabo R. 2008. Botanical knowledge and its differentiation by age, gender and ethnicity in Southwestern Niger. Human Ecology 36:881-889.

Bakhiet A, Adam S. 1995. An estimation of Citrullus colocynthis toxicity for chicks. Veterinary and human toxicology 37:356-358.

Barkaoui M, Katiri A, Boubaker H, Msanda F. 2017. Ethnobotanical survey of medicinal plants used in the traditional treatment of diabetes in Chtouka Ait Baha and Tiznit (Western Anti-Atlas), Morocco. Journal of Ethnopharmacology 198: 338-350.

Bellakhdar J. 1997. La pharmacopée marocaine traditionnelle. Médicine arabe ancienne et savoirs populaires 189.

Bellakhdar J, Claisse R, Fleurentin J, Younos C. 1991. Repertory of standard herbal drugs in the Moroccan pharmacopoea. Journal of Ethnopharmacology 35:123-143.

Bhatia H, Sharma YP, Manhas R, Kumar K. 2014. Ethnomedicinal plants used by the villagers of district Udhampur, J\&K, India. Journal of Ethnopharmacology 151:1005-1018.

Bhatia H, Sharma YP, Manhas R, Kumar K. 2018. Traditionally used wild edible plants of district Udhampur, J\&K, India. Journal of Ethnobiology and Ethnomedicine 14:73.

Broegaard RB, Rasmussen LV, Dawson N, Mertz O, Vongvisouk T, Grogan K. 2017. Wild food collection and nutrition under commercial agriculture expansion in agriculture-forest landscapes. Forest Policy and Economics 84:92-101.

Burlingame B. 2000. Wild nutrition. Journal of Food composition and Analysis 2:99-100

Caniago I, Stephen FS. 1998. Medicinal plant ecology, knowledge and conservation in Kalimantan, Indonesia. Economic Botany 52:229-250.

Chaachouay N, Benkhnigue O, Fadli M, El Ibaoui H, Zidane L. 2019. Ethnobotanical and ethnopharmacological studies of medicinal and aromatic plants used in the treatment of metabolic diseases in the Moroccan Rif. Heliyon 5:e02191.

Diwan F, Abdel Hassan I, Mohammed S. 2000. Effect of saponin on mortality and histopathological changes in mice. EMHJ-Eastern Mediterranean Health Journal 6(2-3):345-351.

DRACS. 2018. La Direction Régionale de l'Agriculture de Casablanca - Settat, El Jadida. https://www.dracs.gov.ma (Accessed 18/06/2019).

Eddouks M, Ajebli M, Hebi M. 2017. Ethnopharmacological survey of medicinal plants used in Daraa-Tafilalet region (Province of Errachidia), Morocco. Journal of Ethnopharmacology 198:516-530.

El Abbouyi PA, Ansari NF, Khyari PSE, Loukili H. 2014. Inventory of medicinal plants prescribed by traditional healers in El Jadida city and suburbs (Morocco). International Journal of Green Pharmacy (IJGP) 8. 
Fakchich J, Elachouri M. 2014. Ethnobotanical survey of medicinal plants used by people in Oriental Morocco to manage various ailments. Journal of Ethnopharmacology 154:76-87.

Fennane M, Tattou MI. 2008. Statistiques et commentaires sur l'inventaire actuel de la flore vasculaire du Maroc. Flora 1986.

Glew RS, Vanderjagt DJ, Chuang L-T, Huang Y-S, Millson M, Glew RH. 2005. Nutrient content of four edible wild plants from West Africa. Plant Foods for Human Nutrition 60:187-193.

Guimbo ID, Mueller JG, Larwanou M. 2011. Ethnobotanical knowledge of men, women and children in rural Niger: a mixed-methods approach. Ethnobotany Research and Applications 9:235-242.

Hadjichambis AC, Paraskeva-Hadjichambi D, Della A, Elena Giusti M, De Pasquale C, Lenzarini C, Censorii E, Reyes Gonzales-Tejero M, Patricia Sanchez-Rojas C, Ramiro-Gutierrez JM. 2008. Wild and semi-domesticated food plant consumption in seven circum-Mediterranean areas. International Journal of Food Sciences and Nutrition 59:383414.

HCP. 2014. Monographie régionale Doukkala-Abda 2014. https://www.hcp.ma/ (Accessed 18/06/2019).

Ickowitz A, Powell B, Rowland D, Jones A, Sunderland T. 2019. Agricultural intensification, dietary diversity, and markets in the global food security narrative. Global Food Security 20:9-16

Hmamouchi M. 1997. Plantes alimentaires, aromatiques, condimentaires, médicinales et toxiques au Maroc. Identification of wild food and non-food plants of the Mediterranean region Chania: CIHEAM-IAMC 89-108

Łuczaj Ł, Končić MZ, Miličević T, Dolina K, Pandža M. 2013. Wild vegetable mixes sold in the markets of Dalmatia (southern Croatia). Journal of Ethnobiology and Ethnomedicine 9:2.

Luczaj L, Pieroni A, Tardío J, Pardo-de-Santayana M, Sõukand R, Svanberg I, Kalle R. 2012. Wild food plant use in 21 st century Europe, the disapperance of old traditions and the search for new ciusines involving wild edibles. Acta Societatis Botanicorum Poloniae 81.

Martin Gj. 1995. Ethnobotany: a methods manual, Chapman y Hall. London, U.K.

Melhaoui A, Jossang A, Bodo B. 1992. Structure of irniine, a pyrrolidine alkaloid from Arisarum vulgare. Journal of Natural Products 55:950-952.

Morales P, Ferreira IC, Carvalho AM, Sánchez-Mata MC, Cámara M, Fernández-Ruiz V, Pardo-de-Santayana M, Tardío J. 2014. Mediterranean non-cultivated vegetables as dietary sources of compounds with antioxidant and biological activity. LWT-Food Science and Technology 55:389-396.

Mrabti HN, Jaradat N, Kachmar MR, Ed-Dra A, Ouahbi A, Cherrah Y, Faouzi MEA. 2019. Integrative herbal treatments of diabetes in Beni Mellal region of Morocco. Journal of Integrative Medicine 17:93-99.

Mugisha MK, Asiimwe S, Namutebi A, Borg-Karlson A-K, Kakudidi EK. 2014. Ethnobotanical study of indigenous knowledge on medicinal and nutritious plants used to manage opportunistic infections associated with HIV/AIDS in western Uganda. Journal of Ethnopharmacology 155: 194-202.

Nassif F, Tanji A. 2013. Gathred food plants in Morocco the long-forgotten species in ethnobotanical research by Fatima Nassif and Abbes Tanji. Life sciences leaflets 37:17-54.

Neves JM, Matos C, Moutinho C, Queiroz G, Gomes LR. 2009. Ethnopharmacological notes about ancient uses of medicinal plants in Trás-os-Montes (northern of Portugal). Journal of Ethnopharmacology 124:270-283.

Powell B, Ouarghidi A, Johns T, Tattou MI, Eyzaguirre P. 2014. Wild leafy vegetable use and knowledge across multiple sites in Morocco: a case study for transmission of local knowledge? Journal of Ethnobiology and Ethnomedicine 10:34.

Rangel CdFCB, Ramos MA, de Amorim ELC, de Albuquerque UP. 2010. A comparison of knowledge about medicinal plants for three rural communities in the semi-arid region of northeast of Brazil. Journal of Ethnopharmacology 127:674-684.

Redouan FZ, Benítez G, Picone RM, Crisafulli A, Yebouk C, Bouhbal M, Driss AB, Kadiri M, Molero-Mesa J, Merzouki A. 2020. Traditional medicinal knowledge of apiaceae at talassemtane national park (northern Morocco). South African Journal of Botany 131:118-130. 
Salhi N, Bouyahya A, Fettach S, Zellou A, Cherrah Y. 2019. Ethnopharmacological study of medicinal plants used in the treatment of skin burns in occidental Morocco (area of Rabat). South African Journal of Botany 121:128-142.

Samant SS, Dhar U. 1997. Diversity, endemism and economic potential of wild edible plants of Indian Himalaya. The International Journal of Sustainable Development \& World Ecology 4:179-191.

Sarfo J, Keding GB, Boedecker J, Pawelzik E, Termote C. 2020. The Impact of Local Agrobiodiversity and Food Interventions on Cost, Nutritional Adequacy, and Affordability of Women and Children's Diet in Northern Kenya: A Modeling Exercise. Frontiers in Nutrition 7:129.

Shrestha PM, Dhillion SS. 2006. Diversity and traditional knowledge concerning wild food species in a locally managed forest in Nepal. Agroforestry Systems 66:55-63.

Silva FdS, Ramos MA, Hanazaki N, Albuquerque UPd. 2011. Dynamics of traditional knowledge of medicinal plants in a rural community in the Brazilian semi-arid region. Revista Brasileira de Farmacognosia 21:382-391.

Tanji A. 2005. Adventices du blé et de l'orge au Maroc. Editions INRA Maroc, Rabat. 458p.

Sukenti K, Hakim L, Indriyani S, Purwanto Y, Matthews PJ. 2016. Ethnobotanical study on local cuisine of the Sasak tribe in Lombok Island, Indonesia. Journal of Ethnic Foods 3:189-200.

Tardío J, Pardo-de-Santayana M. 2008. Cultural importance indices: a comparative analysis based on the useful wild plants of Southern Cantabria (Northern Spain). Economic Botany 62:24-39.

Tbatou M, Belahyan A, Belahsen R. 2016a. Wild edible plants traditionally used in the countryside of El Jadida, coastal area in the Center of Morocco. Life Science Leaflets 75:28-48.

Tbatou M, Belahyan MFA, Belahsen R. 2016b. Wild edible plants traditionally used in the rural area of El Jadida (center of Morocco)-Assessing traditional knowledge erosion by Manal Tbatou1, Mustapha Fagroud, Abdelmonaim Belahyan and Rekia Belahsen. Life Sciences Leaflets 78: 30-51.

Teixidor-Toneu I, Martin GJ, Ouhammou A, Puri RK, Hawkins JA. 2016. An ethnomedicinal survey of a Tashelhitspeaking community in the High Atlas, Morocco. Journal of Ethnopharmacology 188:96-110.

Valdés B. 2002. Catalogue des plantes vasculaires du Nord du Maroc, incluant des clés d'identification. Editorial CSIC-CSIC Press.

Vitalini S, Iriti M, Puricelli C, Ciuchi D, Segale A, Fico G. 2013. Traditional knowledge on medicinal and food plants used in Val San Giacomo (Sondrio, Italy)—An alpine ethnobotanical study. Journal of Ethnopharmacology 145:517529. 